\title{
Si-N-C Nanowires Derived by Polyhydridomethylsilazane Pyrolysis
}

\section{Gongjin Qi}

Beijing Aeronautical Technology Research Center, Beijing, China.

Email: qgjin@tom.com

Received November $10^{\text {th }}, 2010$; revised January $24^{\text {th }}, 2010$; accepted May $19^{\text {th }}, 2011$.

\begin{abstract}
The preceramic polymer, polyhydridomethylsilazane, was synthesized and pyrolyzed at $1873 \mathrm{~K}$ in nitrogen atmosphere to prepare Si- $N-C$ nanowires without using any catalysts. The diameters of the nanowires were from tens of nanometers to several microns, and the maximum length of the nanowires reached several hundred microns. The nanowires had no bulbs or droplets on the tips. However, two different bulbs with diameters of several microns were observed, and one bulb was covered by nanowires all around, while the other bulb had nanowires just covered on its handle.
\end{abstract}

Keywords: Si-N-C Nanowires, Polyhydridomethylsilazane, Pyrolysis

\section{Introduction}

Silicon nitride possesses excellent thermal and mechanical properties and is one of the most important engineering ceramics. Synthesis of one-dimensional nanostructured silicon nitrides has attracted tremendous interest in recent years [1-8], because of their potential in applications where mechanical strength and high-temperature/ corrosive durability are required. What's more, it has been demonstrated that wide-band-gap single-crystalline silicon nitride can be excellent hos material with high doping levels similar to GaN and AlN. The synthesis methods for one-dimensional silicon nitride include chemical vapor deposition process, nitridation of silicon/silica powders or silicon substrates, combustion synthesis, and polymeric precursor pyrolysis method, etc. However, most of the synthesis methods utilize catalysts such as iron, gallium, gallium nitride, and $\mathrm{FeCl}_{2}$.

In our previous paper [9], a preceramic polymer, perhydropolysilazane (PHPS), was used to prepare silicon nitride powders by thermal decomposition. A novel phenomenon was found that silicon nitride-based nanowires appeared on the surface of the polymer-derived ceramic powders although there were no catalysts at all. No bulbs or droplets were observed on the tips of the nanowires, and two gas-solid mechanisms were proposed to explain their growth.

In this paper, we present $\mathrm{Si}-\mathrm{N}-\mathrm{C}$ nanowires derived by another preceramic polymer, polyhydridomethylsilazane (PHMS). The diameters of the nanowires were from tens of nanometers to several microns, and the maximum length reached several hundred microns. The nanowires had no bulbs or droplets on the tips. However, a novel phenomenon was observed that different bulbs with diameters of several microns appeared in addition to the nanowires.

\section{Experimental}

The precursor methylhydridocyclosilazane (MHS) [10], with the structure unit of $\left[\mathrm{CH}_{3} \mathrm{SiHNH}\right]_{\mathrm{n}}$, was synthesized and crosslinked into polyhydridomethylsilazane (PHMS). The polymer PHMS was pyrolyzed at $1873 \mathrm{~K}$ in nitrogen atmosphere (99.99\%) for 2 hours to obtain ceramic nanowires.

The morphology of polymer-derived ceramic powders and nanowires were characterized using field emission scanning electron microscope (FESEM, Sirion 200, FEI Company, Netherlands) equipped with energy dispersive X-ray (EDX) spectrometer (Genesis 60S, EDAX Company, USA).

\section{Results and Discussion}

FESEM micrographs of the PHMS-derived ceramic products are shown in Figure 1. After pyrolysis at 1873 $\mathrm{K}$ in nitrogen atmosphere, the PHMS-derived products were irregular powders with the maximum size of about 500 microns (Figure 1(a)). Among the ceramic products, the surfaces of some powders showed hair-like morphology (Figures 1(b,c)). Magnification of the Fig- 

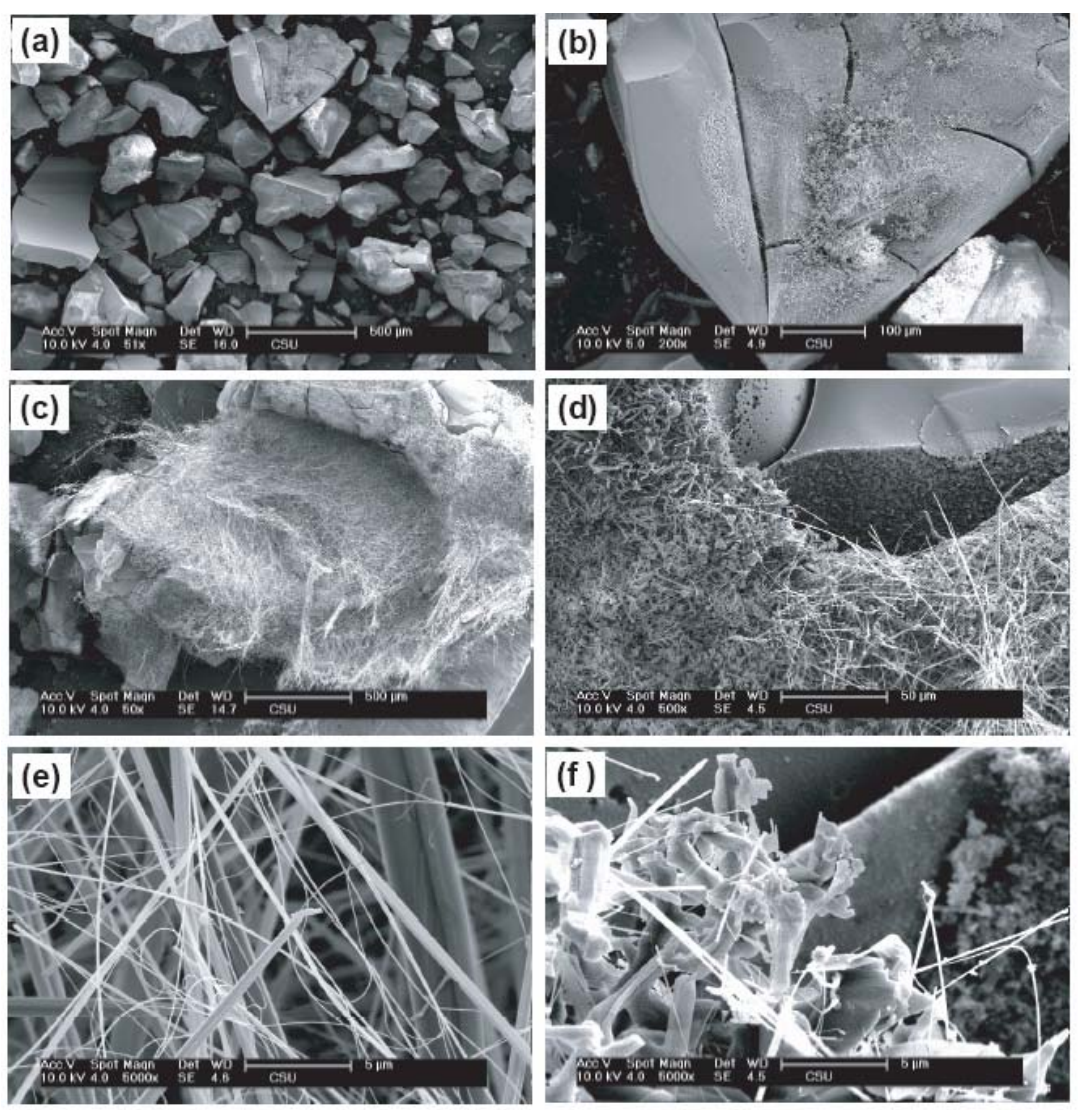

Figure 1. FESEM micrographs of the PHMS-derived ceramic products. (a) micrographs of ceramic powders; (b)(c) nanowires on some powders; (d)(e)(f) magnification of the one-dimensional nanostructures in (c).

ure 1(c) exhibited one-dimensional nanostructures at different sites with different sizes and shapes (Figures 1(d)-(f)). The diameters of the nanowires were from tens of nanometers to several microns, and the maximum length reached several hundred microns. There were no bulbs or droplets on the tips of the nanowires.

In order to determine the composition of the nanowires, EDX spectrum was conducted on the nanowires in Figure 1(c). The composition of the nanowires was silicon, nitrogen and carbon, suggesting that the nanowires were Si-N-C (see Figure 2).

As was seen in Figure 1, the nanowires appeared without bulbs on the tips. In addition to these nanostructures, however, a novel phenomenon arised that few ball-like structures were observed (Figure 3). As shown in Figure 3(a), there was a ball with the diameter of about five microns. Magnification of the ball indicated that the ball was covered by nanowires all around (see Figure 3(b)). Different from Figure 3(a), another bulb structure like a football was found in Figure 3(c), which was a little smaller with the diameter of about three microns. Although there was no nanowires covered on its surface, yet magnification of the bulb indicated that the handle was covered by nanowires in parallel circles (Figure 3(d)).

In Ref. [8], silicon nitride nanowires have been synthesized using a polysilazane pyrolysis method, and $\mathrm{FeCl}_{2}$ catalyst must be used during the process otherwise no nanowires could be obtained. However, in our study, the nanowires were obtained without any catalysts.

Several mechanisms have been proposed for the growth of silicon nitride nanowires, including vaporliquid-solid (VLS), solid-liquid-gas-solid (SLGS), and vapor-solid (VS) mechanisms. As shown in Figure 1, the nanowires had no bulbs or droplets at the tips, suggesting a growth mechanism different from the VLS mechanism. As for the bulbs in Figure 3, it was a just special case. What's more, the sizes of the bulbs were several microns, which had exceeded the size of NANO-wires. The two bulbs in Figure 3 showed different morphology, and they probably had different compositions and different growth mechanisms. In a word, this paper is still a preliminary investigation, and further study on TEM observation and growth mechanism are in progress. 


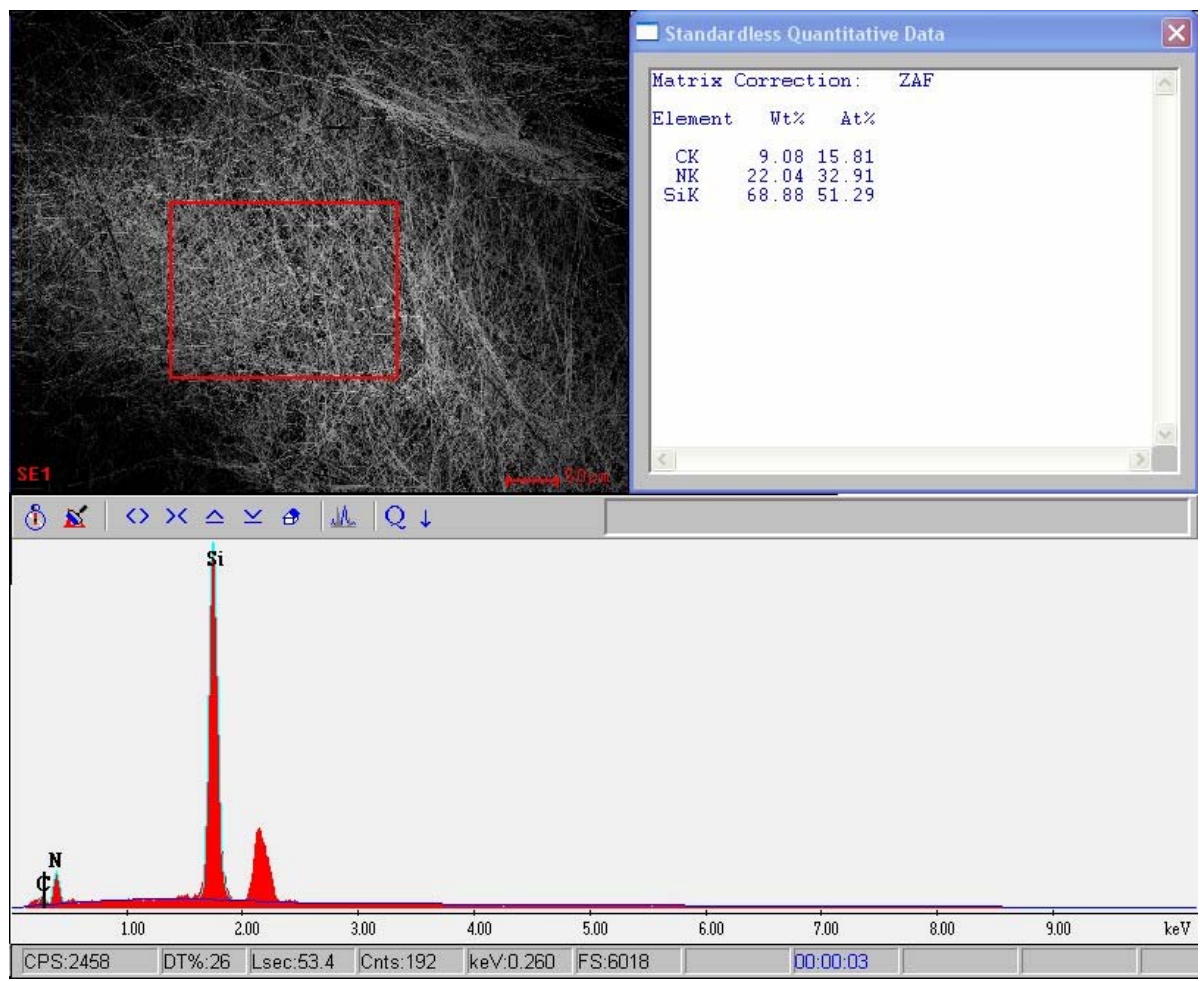

Figure 2. EDX spectrum of the nanowires derived by PHMS pyrolysis.
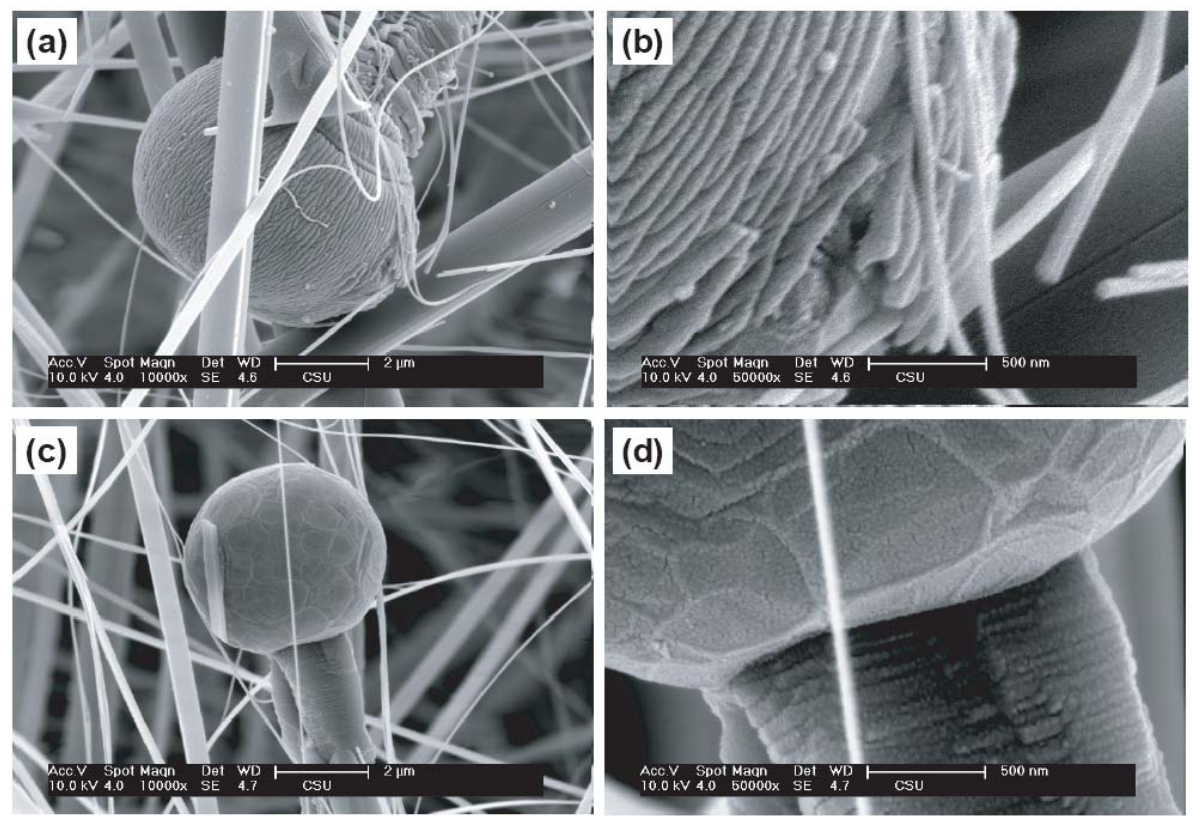

Figure 3. FESEM micrographs of the ball-like structures in PHMS-derived products. (a)(b)ball-like structure covered by nanowires ; (c)(d) football-like structures with the handle covered by nanowires.

\section{Conclusions}

In summary, we have synthesized Si-N-C nanowires by polymeric precursor pyrolysis method using polyhydric- domethylsilazane without any catalysts. The diameters of the nanowires were from tens of nanometers to several microns, and the maximum length reached several hundred microns. There were no bulbs or droplets on the tips 
of the nanowires. In addition to the nanowires, few balllike structures appeared with the diameters of several microns. One bulb was covered by nanowires all around, while the other bulb just had nanowires covered on its handle.

\section{REFERENCES}

[1] X. C. Wu, W. H. Song, B. Zhao, et al., "Synthesis of Coaxial Nanowires of Silicon Nitride Sheathed with Silicon and Silicon Oxide," Solid State Communications, Vol. 115 , No. 12, 2000, pp. 683-686. doi:10.1016/S0038-1098(00)00255-6

[2] C. Rath, A. Pinyol, J. Farjas, et al., "Si-N Nanowire Formation from Silicon Nano and Microparticles," Materials Research Society Symposium-Proceedings, Vol. 789, 2004, pp. 1-4.

[3] H. Cui and B. R. Stoner, "Nucleation and Growth of Silicon Nitride Nanoneedles Using Microwave Plasma Heating," Journal of Material Research, Vol. 16, No. 11, 2001, pp. 3111-3115. doi:10.1557/JMR.2001.0429

[4] Y. Zhang, N. Wang, R. He, et al., "A Simple Method to Synthesize $\mathrm{Si}_{3} \mathrm{~N}_{4}$ and $\mathrm{SiO}_{2}$ Nanowires from $\mathrm{Si}$ or $\mathrm{Si} / \mathrm{SiO}_{2}$ Mixture," Journal of Crystal Growth, Vol. 233, No. 4, 2001, pp. 803-808. doi:10.1016/S0022-0248(01)01650-5

[5] H. Y. Kim, J. Park and H. Yang, "Synthesis of Silicon
Nitride Nanowires Directly from the Silicon Substrates," Chemical Physics Letters, Vol. 372, No. 1-2, 2003, pp. 269-274. doi:10.1016/S0009-2614(03)00428-7

[6] C. Xu, M. Kim, J. Chun, et al., "Gallium-Doped Silicon Nitride Nanowires Sheathed with Amorphous Silicon Oxynitride," Scripta Materialia, Vol. 53, No. 8, 2005, pp. 949-954. doi:10.1016/j.scriptamat.2005.06.024

[7] H. Chen, Y. Cao, X. Xiang, et al., "Fabrication of $\beta-\mathrm{Si}_{3} \mathrm{~N}_{4}$ Nano-Fibers," Journal of Alloys and Compounds, Vol. 325, No. 1-2, 2001, pp. L1-L3. doi:10.1016/S0925-8388(01)01383-4

[8] W. Yang, Z. Xie, J. Li, et al., "Ultra-Long Single-Crystalline $\alpha-\mathrm{Si}_{3} \mathrm{~N}_{4}$ Nanowires: Derived from a Polymeric Precursor," Journal of the American Ceramic Society, Vol. 88, No. 6, 2005, pp. 1647-1650. doi:10.1111/j.1551-2916.2005.00270.x

[9] G. J. Qi, C. R. Zhang and H. F. Hu, "Synthesis of Silicon Nitride Nanowires by the Pyrolysis of Perhydropolysilazane," Journal of Nanoscience and Nanotechnology, Vol. 6, No. 5, 2006, pp. 1486-1488. doi:10.1166/jnn.2006.306

[10] D. Seyferth and G. H.Wiseman, "High-Yield Synthesis of $\mathrm{Si}_{3} \mathrm{~N}_{4} / \mathrm{SiC}$ Ceramic Materials by Pyrolysis of a Novel Polyorganosilazane," Communications of the American Society, Vol. 7, 1984, pp. C132-133. 